\title{
Potential probiotic of Lactobacillus johnsonii LT171 for chicken nutrition
}

\author{
Hamidreza Taheri ${ }^{1 *}$, Fatemeh Tabandeh ${ }^{2}$, Hossein Moravej ${ }^{1}$, Mojtaba Zaghari ${ }^{1}$, Mahmood \\ Shivazad ${ }^{1}$ and Parvin Shariati ${ }^{2}$
}

Department of Animal Science, University College of Agriculture and Natural Resources, University of Tehran, 3158711167, Karaj, Iran.

${ }^{2}$ Industrial and Environmental Biotechnology Department, National Institute of Genetic Engineering and Biotechnology (NIGEB), 14965-161, Tehran, Iran.

Accepted 14 September, 2009

\begin{abstract}
The objective of this research was to investigate the potential probiotic of Lactobacillus johnsonii LT171. It had aggregation $(60 \mathrm{~min})$ and antibacterial effects against Salmonella Enteritidis, Salmonella Typhimurium and Escherichia coli 078:K80. It showed amylase and protease activity and high clear zone in culture medium containing calcium phytate; cell surface hydrophobicity, $85.21 \pm 7.27 \%$; resistance to acidic condition (pH 3 for $90 \mathrm{~min}$ ) and bile salts (in culture medium containing $0.075 \%$ ox gall). Also it had resistance to nalidixic acid and neomycine. This research showed appropriate probiotic properties of $L$. johnsonii LT171 for chicken nutrition. Hence this strain can complement the characteristics of other strains in multistrain probiotics because of its high clear zone in culture medium containing calcium phytate.
\end{abstract}

Key words: Lactobacillus johnsonii LT171, probiotic, chicken.

\section{INTRODUCTION}

The important role of gastrointestinal microflora in the health and nutrition of animals and humans is increasingly recognized. Probiotics are defined as live microbial food supplements that beneficially affect the host by improving intestinal microbial balance (Fuller, 1989). Among the potential probiotics, lactic acid bacteria (LAB) is reported to have important effects on animal performance (Chou and Weimer, 1999).

One of the most reported lactic acid bacteria as a source of chicken probiotic is Lactobacillus johnsonii. La Ragione et al. (2004) and Van Coillie et al. (2007) examined the ability of $L$. johnsonii to prevent the colonization of pathogens in chicken. On the other hand, $L$. johnsonii belongs to the group of obligatory homofermentative bacteria that have high total antioxidative activity. This group of Lactobacilli was found to have a relatively much stronger inhibitory activity in an anaerobic environment and has also been shown to have no

*Corresponding author. E -mail: taherihr@gmail.com. Tel: +98 261 2248082. Fax: +982612246752. inhibitory activity toward any species or isolate of the genus Lactobacillus (Annuk et al., 2003). Hence this research was conducted to investigate the most important properties of $L$. johnsonii LT171, an indegenus poultry-derived isolate, for potential probiotic. Adhesion ability to the mucus and antibacterial effects against pathogens is the major criteria in the selection of $L A B$ for probiotic candidates (Garriga et al., 1998; Ehrmann et al., 2002). The adherence capability of a bacterial strain to the digestive tract is presumably a prerequisite for its colonization (Bouzaine et al., 2005). These attributes (adhesion ability and antibacterial effects) of LAB provide competitive exclusion against enteric pathogens and reduce their colonization. Competitive exclusion concept was applied first to the domestic fowl by Nurmi and Rantala (1973) when they attempted to control a severe outbreak of Salmonella Infantis in Finnish broiler flock. Hence, the examination of LAB ability for this concept has been done in many studies (Edens et al., 2007; La Ragion et al., 2004).

Since $L A B$ can reduce the number of pathogens in the gastrointestinal tract by production of bacteriocins and especially organic acids, the antibacterial effects of $L$. 
johnsonii LT171 were investigated in this research. The tests of aggregation and cell surface hydrophobicity are representative for adhesion ability of $L A B$ to the mucus, indirectly (Garriga et al., 1998; Ehrmann et al., 2002; Taheri et al., 2009) and co-aggregation is thought to be linked to the ability to interact closely with undesirable bacteria (Gusils et al., 1999). These attributes are another characteristics representing competitive exclusion of this strain against enteric pathogens. Therefore, they were examined as well. On the other hand, recent studies have focused to improve the probiotic efficacy and represent more applicable supplements in poultry nutrition. The beneficial effects of expression of $\alpha$ amylase, phytase, $\beta$-glucanase, xylanase and cellulase enzymes in the Lactobacillus (Scheirlinck et al., 1990; Liu et al., 2005, 2007; Yu et al., 2008) show the importance of enzymatic activities in bacterial strains of chicken probiotics. A probiotic that has the enzymatic activities can improve digestion especially in newly-hatched chicks. Hence, the enzymatic activities of this strain were also examined. Resistance to $\mathrm{pH}$ and bile salts is of great importance in survival and growth of bacteria in the intestinal tract and thus, is a prerequisite for choosing suitable probiotics. Tolerance to acidic condition and bile salts of $L$. johnsonii LT171 were investigated in this study as well as other tests.

\section{MATERIAL AND METHODS}

\section{Chemicals}

All chemicals used in this study were of analytical grade, mostly purchased from Sigma Chemical Co., St. Louis, MO, USA. MRS (De Man, Rogosa and Sharpe) and nutrient broth media were purchased from Merck, Darmstadt, Germany and Oxoid Ltd., Basingstoke, UK, respectively.

\section{Isolation of bacterial strain}

L. johnsonii LT171 was isolated from digestive tract (ileum) of broiler chicken and identified by sequencing the 16S rRNA. Three strains of avian origin, Salmonella Enteritidis, Salmonella Typhimurium and Escherichia coli O78:K80 were obtained from the Veterinary Department at the University of Tehran, Iran. They were used examining antagonistic activity and co-aggregation behavior of L. johnsonii LT171.

\section{Detection of enzymatic activities}

Enzyme (that is, amylase, protease, lipase and phytase) activities were conducted according to Taheri et al. (2009). In order to detect the amylase, lipase and phytase activities, the strain was subcultured and then spot-inoculated onto relevant medium, which was incubated anaerobically (in anaerobic jars; Sharif Azmayeshgahi Co., Tehran, Iran) for $48 \mathrm{~h}$ at $37^{\circ} \mathrm{C}$. After incubation, the halo zone surrounding colony was measured with a caliper. For amylase activity, the strain was sub-cultured on mMRS (modified MRS: MRS prepared in the lab, with $0.25 \%$ starch instead of whole glucose) broth. Activity was examined using a medium that consisted of meat peptone $(0.5 \%)$, yeast extract $(0.7 \%), \mathrm{NaCl}(0.2 \%)$, starch (2\%) and agar (1.5\%). For detecting the clear zones of amylase activity, dense Lugol's solution was poured over the plates. In the case of lipase activity, the MRS broth containing olive oil (1\%) and Arabic gum $(1 \%)$ was used to sub-culture the strain. Activity was detected by using a medium that consisted of tryptone $(0.1 \%)$, yeast extract $(0.5 \%), \mathrm{NaCl}(0.05 \%)$, olive oil $(0.1,0.5$ or $1 \%)$, Arabic gum $(1 \%)$ and agar (1.5\%).

The MRS broth which contained $0.25 \%$ calcium phytate (SigmaAldrich Co., St. Louis, MO, USA) was used to sub-culture the strain and the medium consisted of glucose $(1.5 \%)$, calcium phytate $(0.5 \%), \quad \mathrm{NH}_{4} \mathrm{NO}_{3}(0.5 \%), \quad \mathrm{KCl}(0.05 \%), \mathrm{MgSO}_{4} .7 \mathrm{H}_{2} \mathrm{O} \quad(0.05 \%)$, $\mathrm{MnSO}_{4} .7 \mathrm{H}_{2} \mathrm{O}(0.02 \%), \mathrm{FeSO}_{4} .7 \mathrm{H}_{2} \mathrm{O}(0.001 \%)$ and agar $(1.5 \%)$ (adjusted to $\mathrm{pH} 7.0$ by $\mathrm{Ca}(\mathrm{OH})_{2}$ ) was used for detection of clear zone.

For detection of protease activity, the strain was cultured on MRS broth and after anaerobic incubation for $24 \mathrm{~h}$ at $37^{\circ} \mathrm{C}, 30 \mu \mathrm{l}$ of culture supernatant was transfered onto a disc placed over a medium consisting of skim milk (1\%) and agar (1.5\%). Clear zones surrounding disc was measured with a caliper.

\section{Detection of antagonistic activity}

For detection of inhibitory activity, the well diffusion assay described by Schillinger and Lucke (1989) was used. Plates containing solidified nutrient agar $(20 \mathrm{ml})$ overlaid with $10 \mathrm{ml}$ of soft nutrient agar $(0.7 \%$ agar in nutrient broth) were inoculated with $5 \mu \mathrm{l}$ of an overnight culture of $S$. Enteritidis, $S$. Typhimurium or E. coli O78: K80. Wells were made in the agar and $30 \mu \mathrm{l}$ of the culture supernatant of $L$. johnsonii LT171 was transferred into the well. The plates were incubated aerobically for $24 \mathrm{~h}$ at $37^{\circ} \mathrm{C}$, after which they were examined for clear inhibition zones around the wells. The test was carried out two times with duplicates each time. This test was evaluated by the disc diffusion assay as well. The procedure was the same as described before except that the paper discs (Tadbir Fan Azma Co., Tehran, Iran) were used instead of the wells.

\section{Aggregation test}

The test was performed by the method of Reniero et al. (1992). Aggregation was scored positive when bacteria gravitated to the bottom of the tubes, leaving a clear supernatant fluid. The test was examined every 15 min for $2 \mathrm{~h}$.

\section{Cell surface hydrophobicity test}

Cell surface hydrophobicity was determined by the method of Rosenberg et al. (1980). The strain was harvested after $18 \mathrm{~h}$ of growth, washed twice and resuspended in physiological saline solution to an optical density (OD) of 0.5 at $600 \mathrm{~nm}$. To test tubes containing $3 \mathrm{ml}$ of washed cells, $1 \mathrm{ml}$ of toluene was added. The mixtures were blended on a vortex for $90 \mathrm{~s}$. The tube was left to stand for $15 \mathrm{~min}$ for separation of the two phases and the $\mathrm{OD}_{600}$ of the aqueous phase was then measured. Hydrophobicity was calculated as the percentage decrease in the $O D_{600}$ of the bacterial suspension due to partitioning of cells into the hydrocarbon layer. Percentage of hydrophobicity $=\left[\left(\mathrm{OD}_{600}\right.\right.$ before mixing $-\mathrm{OD}_{600}$ after mixing) / $O D_{600}$ before mixing] $\times 100$.

\section{Co-aggregation test}

Reid et al. (1988) suggested that the dominance of inhibitorproducing lactobacilli on the urogenital epithelium of humans and the ability of these organisms to interact closely with uropathogens such as E. coli would constitute an important host defense mecha- 
Table 1. Different attributes of L. johnsonii LT171 (this research) in compare to L. crispatus LT116 (a candidate strain as a source of probiotic; Taheri et al. 2009).

\begin{tabular}{|l|c|c|}
\hline \multicolumn{1}{|c|}{ Parameter } & \multicolumn{2}{c|}{ Strains } \\
\cline { 2 - 3 } & L. johnsonii LT171 & L. crispatus LT116 \\
\hline Antibacterial activity & 1.2 & 0.91 \\
Amylase activity & $+^{2}$ & +++ \\
Clear zone in culture medium containing calcium phytate & +++ & + \\
Protease activity (mm) & $15.05 \pm 2.75$ & $14.2 \pm 2.54$ \\
Lipase activity $_{\text {Aggregation time }^{3} \text { (min) }}$ & - & - \\
Cell surface hydrophobicity (\%) $^{*}$ & 60 & 15 \\
\hline
\end{tabular}

${ }^{1}$ The mean of growth inhibition radius $(\mathrm{mm})$ against all pathogens ( $S$. Enteritidis, S. Typhimurium and E. coli O78:K80).

${ }_{+++}$, Halo zone was more than $3 \mathrm{~mm} ;+$, halo zone was just detected as less than one $\mathrm{mm}$

${ }^{3}$ Time needed to give a clear supernatant fluid, lower aggregation time indicates more aggregation of each strain.

nism against infection. Hence co-aggregation was investigated according to Jin et al. (1998). Suspension of the strain, E. coli O78: $\mathrm{K} 80, S$. Enteritidis or $S$. Typhimurium was adjusted in phosphate buffer $(\mathrm{pH} 7)$ to an $\mathrm{OD}_{600}$ of 0.5 . A suspension $(0.5 \mathrm{ml})$ of each pathogen and a similar suspension $(0.5 \mathrm{ml})$ of the strain was placed together in a test tube and mixed thoroughly using a vortex. The $\mathrm{OD}_{600}$ of the bacterial mixture was measured after incubation for $4 \mathrm{~h}$ at $37^{\circ} \mathrm{C}$. Control tubes contained $1 \mathrm{ml}$ of a suspension of each bacterial species. The percentage of co-aggregation was calculated using the equation of Handly et al. (1987):

Percentage of co-aggregation $=\{[(\mathrm{PC}+\mathrm{LC}) / 2-(\mathrm{P}+\mathrm{L})] /(\mathrm{PC}+\mathrm{LC}) / 2\}$ $\times 100$

where PC and LC represent the optical densities in control tubes containing only pathogen or Lactobacillus after $4 \mathrm{~h}$ of incubation, respectively; $\mathrm{P}+\mathrm{L}$ represent the optical density of the mixed culture after the same period of incubation.

\section{Bile salts tolerance test}

Overnight culture of the strain was centrifuged (Beckman, J-6M; Beckman Coulter, Fullerton, CA, USA) at $7500 \mathrm{~g}$ for $5 \mathrm{~min}$ at $4^{\circ} \mathrm{C}$. After re-suspending the pellets in the phosphate buffer $(\mathrm{pH} \mathrm{6})$, it was diluted $1 \times 10^{-5}$. Subsequently, the counts of viable cells were determined by growing the suspensions on MRS containing 0.075 , $0.15,0.3$ and $1 \%(w / v)$ ox-bile (Fluka Biochemika, Sigma-Aldrich, Buchs, Switzerland) anaerobically at $37^{\circ} \mathrm{C}$ for $48 \mathrm{~h}$ (Garriga et al., 1998).

\section{Acidic $\mathrm{pH}$ tolerance test}

Cell suspension was prepared as above and then diluted $1 \times 10^{-5}$ in phosphate buffer at $\mathrm{pH} 3$ and 6 . The suspensions were then incubated for $90 \mathrm{~min}$ at $37^{\circ} \mathrm{C}$. The viable cells were then counted by growing the suspensions on MRS agar anaerobically at $37^{\circ} \mathrm{C}$ for 48 h (Garriga et al., 1998).

\section{Antibiogram test}

Antibiotic sensitivity of the strain was determined as follow: the density of bacterial suspension was adjusted until the visible turbidity was equal to $0.5 \mathrm{McF}$ arland standard. The inoculum was spread evenly over the entire surface of the plates contained MRS agar. Subsequently, paper discs containing the antibiotics (Tadbir Fan Azma Co, Tehran, Iran) were laid on the plates and incubated anaerobically at $37^{\circ} \mathrm{C}$ and growth inhibition was examined after 24 h.

\section{RESULTS AND DISCUSSION}

We have earlier screened 332 LAB based on aggregation time, antibacterial effects, enzymatic activity, cell surface hydrophobicity, co-aggregation, tolerance to bile salts and acidic condition and finally selected Lactobacillus crispatus LT116 as a source of chicken probiotic because of its predominant characteristics in comparison to the other isolated strains from the gastrointestinal tract of chickens (Taheri et al., 2009). We reported the enzymatic results of LAB as "nd", not detected (without enzymatic activity); "+", halo zone was just detected as less than one mm (low enzymatic activity) and "+++", halo zone was more than $3 \mathrm{~mm}$ that was noticeable compared to other strains (high enzymatic activity). Since all methods used in this study was similar to their research, the results of L. johnsonii LT171 are represented in comparison with their findings (Table 1 ). The results showed that L. johnsonii LT171 had no extra lipase production; however, it showed low amylase activity and high clear zone in culture medium containing calcium phytate. Although Sreeramulu et al. (1996) and Zamudio et al. (2001) showed the high phytase activity of Lactobacillus plantarum and L. amylovorus among Lactobacillus, respectively, they did not examine the phytase activity of $L$. johnsonii in their studies. Halo zone for protease activity was $15.05 \pm 2.75 \mathrm{~mm}$. L. johnsonii LT171 had antibacterial effect against all pathogens ( $S$. Enteritidis, $S$. Typhimurium and E. coli O78:K80). Also it had aggregation, $60 \mathrm{~min}$ and cell surface hydrophobicity, $85.21 \pm$ $7.27 \%$.

Aggregation shows clumping of strains together and also adhesion ability to the epithelial cells indirectly but in a strong way (Garriga et al., 1998; Ehrmann et al., 2002; Taheri et al., 2009). It has been reported that bacteria 
Table 2. Antibiogram profile of L. johnsonii LT171.

\begin{tabular}{|l|c|}
\hline \multicolumn{1}{|c|}{ Antibiotic } & Halo zone $(\mathbf{m m})$ \\
\hline Ampicillin $(10 \mu \mathrm{g})$ & 22 \\
Bacitracin $(10 \mathrm{units})$ & 30 \\
Chloramphenicol $(30 \mu \mathrm{g})$ & 30 \\
Doxycycline $(30 \mu \mathrm{g})$ & 6 \\
Erythromycin $(15 \mu \mathrm{g})$ & 25 \\
Furazolidon $(100 \mu \mathrm{g})$ & 7 \\
Florfenikol $(30 \mu \mathrm{g})$ & 28 \\
Gentamicin $(10 \mu \mathrm{g})$ & 14 \\
Kanamycin $(30 \mu \mathrm{g})$ & 24 \\
Lincomycin $(2 \mu \mathrm{g})$ & 3 \\
Nalidixic acid $(30 \mu \mathrm{g})$ & 0 \\
Neomycine $(30 \mu \mathrm{g})$ & 0 \\
Oxytetracyclin $(30 \mu \mathrm{g})$ & 6 \\
Penicillin $(10 \mathrm{units})$ & 28 \\
Streptomycin $(10 \mu \mathrm{g})$ & 12 \\
Tetracycline $(30 \mu \mathrm{g})$ & 3 \\
Vancomycin $(30 \mu \mathrm{g})$ & 20 \\
\hline
\end{tabular}

which show high aggregation (or in other words, low aggregation time), also have high cell surface hydrophobicity and adhesion ability to the mucus (Taheri et al., 2009).

Aggregation and cell surface hydrophobicity of the bacteria could be used instead of the examination of adhesion ability to the mucus (Taheri et al., 2009), because there is a strong relationship among these characteristics especially between aggregation time and adhesion ability to the epithelium of the digestive tract (Garriga et al., 1998). The results of this study showed no co-aggregation between L. johnsonii LT171 and enteric strains. Jin et al. (1998) showed that there was poor co-aggregation between selected LAB and E. coli strains. These authors indicated that most isolated bacteria had co-aggregation of between 1 to $4.6 \%$. With regard to the $\mathrm{pH}$ of feed in the gastrointestinal tract of the chicken (Church and Pond, 1974), it was thus preferred to examine the survivability of $L$. johnsonii LT171 at pH 3 for 90 min. This strain was completely resistance to this $\mathrm{pH}$. Garriga et al. (1998) screened LAB with regard to their tolerance to $\mathrm{pH} 3$ and they showed that $\mathrm{pH} 3$ did not decrease the number of $L A B$. The effects of bile salts on the survival of lactobacilli have been investigated by several authors and are thought to be linked to the ability to de-conjugate bile acids (Tannock et al., 1997). L. johnsonii LT171 showed resistance in culture medium containing $0.075 \%$ bile salts. Antibiogram profile is also important, since many antibacterial chemicals are used as feed additives in poultry farms. There is no information about minimum inhibitory concentration break points for Lactobacillus in the NCCLS (data is only for pathogenic bacteria), hence only the size of halo zone of $L$. johnsonii LT171 is represented in Table 2. There was not any growth inhibition against this strain in nalidixic acid and neomycine antibiotics. In general, L. johnsonii LT171 had appropriate probiotic properties. This strain can complement the characteristics of other strains in multistrain probiotics and be more effective in such supplements. Timmerman et al. (2004) showed that multistrain or multispecies probiotics were more effective than monostrain probiotics for resistance to pathogens and performance in animals. Since our previous results (Taheri et al., 2009) showed that $L$. crispatus LT116 (a candidate strain) had low clear zone in culture medium containing calcium phytate, $L$. johnsonii LT171 with high activity of this attribute can be useful in probiotic that contained L. crispatus LT116 plus $L$. johnsonii LT171. Noticeable characteristics of $L$. crispatus LT116 are high amylase activity and aggregation (Taheri et. al., 2009) and of L. johnsonii LT171 is high clear zone in culture medium containing calcium phytate. Hence these strains can complement the characteristics of each other in a doublestrain probiotic.

A separate study will be required for the evaluation of L. johnsonii LT171 and L. crispatus LT116, alone or in combination, as a probiotic supplement on chicken performance.

\section{ACKNOWLEDGEMENT}

This work was financially supported by the Iranian National Science Foundation (INSF).

\section{REFERENCES}

Annuk H, Shchepetova J, Kullisaar T, Songisepp E, Zilmer M, Mikelsaar M (2003). Characterization of intestinal Lactobacilli as putative probiotic candidates. J. Appl. Microbiol. 94: 403-412.

Bouzaine T, Dauphin RD, Thonart P, Urdaci MC, Hamdi M (2005). Adherence and colonization properties of Lactobacillus rhamnosus TB1, a broiler chicken isolate. Lett. Appl. Microbiol. 40: 391-396.

Chou LS, Weimer B (1999). Isolation and characterization of acid- and bile-tolerant isolates from strains of Lactobacillus acidophilus. J. Dairy Sci. 82: 23-31.

Church DC, Pond WG (1974). The gastrointestinal tract and nutrition. In Basic Animal Nutrition and Feeding. Albany Printing. Albany, New York. pp. 25-49

Edens FW, Parkhurst CR, Casas IA, Dobrogosz WJ (1997). Principles of ex ovo competitive exclusion and in ovo administration of Lactobacillus reuteri. Poult. Sci. 76: 179-196.

Fuller R (1989). Probiotics in man and animals. J. Appl. Bacteriol. 66: 365-378.

Garriga M, Pascual M, Monfort JM, Hugas M (1998). Selection of lactobacilli for chicken probiotic adjuncts. J. Appl. Microbiol. 84: 125132.

Gusils C, Perez Chaia A, Gonzalez S, Oliver G (1999). Lactobacilli isolated from chicken intestines: potential use as probiotics. J. Food Prot. 62: 252-256.

Handly PS, Harty DWS, Wyatt JE, Brown CR, Doran JP, Gibbs ACC (1987). A comparison of the adhesion, co-aggregation and cellsurface hydrophobicity properties of fibrillar and fimbriate strains of Streptococcus salivarius. J. Gen. Microbiol. 133: 3207-3217.

Jin LZ, Ho YW, Abdullah N, Ali MA, Jalaludin S (1998). Note: lack of influence of adherent Lactobacillus isolates on the attachment of Escherichia coli to the intestinal epithelial cells of chicken in vitro. J. Appl. Microbiol. 84: 1171-1174. 
La Ragione RM, Narbad A, Gasson MJ, Woodward MJ (2004). In vivo characterization of Lactobacillus johnsonii Fl9785 for use as a defined competitive exclusion agent against bacterial pathogens in poultry. Lett. Appl. Microbiol. 38: 197-205.

Liu JR, Yu B, Liu FH, Cheng KJ, Zhao X (2005). Expression of Rumen Microbial Fibrolytic Enzyme Genes in Probiotic Lactobacillus reuteri. Appl. Environ. Microbiol. 71: 6769-6775.

Liu JR, Lai SF, Yu B (2007). Evaluation of an intestinal Lactobacillus reuteri strain expressing rumen fungal xylanase as a probiotic for broiler chickens fed on a wheat-based diet. Br. Poult. Sci. 48: 507514.

Nurmi E, Rantala M (1973). New aspects of Salmonella infection in broiler production. Nature, 241: 210-211.

Reid G, McGroarty GA, Angotti R, Cook RL (1988). Lactobacillus inhibitor production against Escherichia coli and coaggregation ability with uropathogens. Can. J. Microbiol. 34: 344-351.

Reniero R, Cocconcelli PS, Bottazzi V, Morelli L (1992). High frequency of conjugation in Lactobacillus mediated by an aggregation-promoting factor. J. Gen. Microbiol. 138: 763-768.

Rosenberg M, Gutnick D, Rosenberg E (1980). Adherence of bacteria to hydrocarbons: a simple method for measuring cell-surface hydrophobicity. FEMS Microbiol. Lett. 44: 929-937.

Scheirlinck T, Mahillion J, Joos H, Dhaese P, Michiels F (1990). Integration and expression of $\alpha$-amylase and endoglucanase genes in the Lactobacillus plantarum chromosome. Appl. Environ. Microbiol. 55: $2130-2137$.

Schillinger U, Lucke F (1989). Antibacterial activity of Lactobacillus sake isolated from meat. Appl. Environ. Microbiol. 55: 1901-1906.
Sreeramulu G, Srinivasa DS, Nand K, Joseph R (1996). Laciobacillus amylovorus as a phytase producer in submerged culture. Lett. Appl. Microbiol. 23: 385-388.

Taheri HR, Moravej H, Tabandeh F, Zaghari M, Shivazad M (2009). Screening of lactic acid bacteria toward their selection as a source of chicken probiotic. Poult. Sci. 88:1586-1593.

Tannock GW, Bateup JM, Jenkinson HF (1997). Effect of sodium taurocholate on the in vitro growth of Lactobacilli. Microb. Ecol. 33: 163-167.

Timmerman HM, Koningb CJM, Mulderc L, Romboutsd FM, Beynen AC (2004). Monostrain, multistrain and multispecies probiotics-A comparison of functionality and efficacy. Int. J. Food Microbiol. 96: 219-233.

Van Coillie E, Goris J, Cleenwerck I, Grijspeerdt K, Botteldoorn N, Van Immerseel F, De Buck J, Vancanneyt M, Swings J, Herman L, Heyndrickx M (2007). Identification of lactobacilli isolated from the cloaca and vagina of laying hens and characterization for potential use as probiotics to control Salmonella Enteritidis. J. Appl. Microbiol. 102: $1095-1106$.

Yu B, Liu JR, Hsiao FS, Chiou PWS (2008). Evaluation of Lactobacillus reuteri $\mathrm{Pg} 4$ strain expressing heterologous $\beta$-glucanase as a probiotic in a barley-base poultry diet. Anim. Feed Sci. Tech. 141: 82-91.

Zamudio M, Gonza'lez A, Medina JA (2001). Lactobacillus plantarum phytase activity is due to non-specific acid phosphatase. Lett. Appl. Microbiol. 32: 181-184. 